\title{
Cochlear implantation on patients with bilateral deafness caused by otitis media with ANCA-associated vasculitis (OMAAV): reports of four cases
}

Takeshi Watanabe, M.D.Ph.D. ${ }^{1)}$, Naohiro Yoshida, M.D.Ph.D. ${ }^{2)}$,

Kan Kishibe, M.D. Ph.D. ${ }^{3)}$, Yuka Morita, M.D.Ph.D.4),

Haruo Takahashi, M.D. Ph.D. $\left.{ }^{1}\right)$, Yasuaki Harabuchi, M.D. Ph.D. ${ }^{3)}$

1) Department of Otolaryngology - Head and Neck Surgery,

Nagasaki University Graduate School of Biomedical Sciences

2) Department of Otolaryngology-Head and Neck Surgery,

Jichi University Saitama Medical Center

3) Department of Otolaryngology-Head and Neck Surgery,

Asahikawa Medical University

4) Department of Otolaryngology-Head and Neck Surgery,

Niigata University

Keywords: Antineutrophil cytoplasmic antibody (ANCA), cochlear implantation, otitis media 


\begin{abstract}
Objective: Antineutrophil cytoplasmic antibody (ANCA)-associated vasculitis (AAV) without systemic symptoms but with initial symptoms related to the ear, such as hearing loss, otalgia, and dizziness, has recently been reported. We have categorized this condition as otitis media with AAV (OMAAV), and have recently proposed its diagnostic criteria.
\end{abstract}

Methods: To determine the effectiveness of cochlear implantation (CI) in patients with profound hearing loss due to OMAAV. We examined the language understanding ability of four patients with bilateral profound or total deafness due to OMAAV, who underwent CI.

Results: In three of the four patients, the language understanding ability with CI was poor. These three patients with poor performance had characteristic features, including a short interval from the onset of ear symptoms to total deafness and clear enhancement of the cochlea on magnetic resonance imaging $(\mathrm{MRI})$.

Conclusion: The poor results observed in patients with a rapidly progressive history of hearing loss were attributed to possible severe and profuse intracochlear bleeding and/or destruction of structures, including the spiral 
ganglion. All the three patients showed contrast enhancement in the inner ear on MRI. We believe that preoperative evaluation of the history of hearing loss as well as the findings of contrast-enhanced MRI is important for predicting the prognosis after CI.

Keywords: antineutrophil cytoplasmic antibody (ANCA), cochlear implantation (CI), otitis media with antineutrophil cytoplasmic antibody-associated vasculitis (OMAAV) 


\section{Introduction}

Antineutrophil cytoplasmic antibody (ANCA)-associated vasculitis (AAV) is characterized by systemic necrotizing vasculitis. Although AAV is commonly accompanied by systemic symptoms particularly in the upper respiratory tract, lungs, and kidneys, AAV without systemic symptoms but with initial symptoms related to ear, such as hearing loss, otalgia, and dizziness, has recently been reported.1-3) We have categorized this condition as otitis media with AAV (OMAAV), and have recently proposed its diagnostic

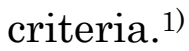

Definition of OMAAV is as follows, if the following three criteria (A, B, C) are fulfilled: (A) disease onset with initial signs/symptoms due to intractable otitis media with effusion or granulation, which is resistant to antibiotics and insertion of tympanic ventilation tubes; (B) at least one of the following three findings: (1) positivity for serum MPO- or PR3-ANCA; (2) histopathology consistent with AAV; and (3) at least one accompanying sign/symptom of AAV-related involvement other than the ear; and (C) exclusion of the other types of intractable otitis media. ${ }^{1)}$ 
OMAAV is an intractable otitis media, which requires a considerable amount of time to be diagnosed as well as to start a specific therapy, including medication, and has a poor prognosis in terms of hearing. ${ }^{4)}$ Recently, owing to early detection and intervention using therapies such as steroid or immunosuppressive agents, remarkable recovery has been observed in approximately $40 \%$ of such patients, and another $30 \%$ patients have shown moderate recovery. In the remaining $30 \%$, however, no improvement or even worsening of hearing was observed, and once they become totally deaf, hearing recovery was reported to be difficult 1,2,4)

Here we report four patients with bilateral profound or total deafness due to OMAAV, who underwent cochlear implantation (CI).

\section{Case presentation}

Case 1: 71-year-old female

The patient had a history of recurrent otitis media in her childhood.

Five months before visiting our hospital, she experienced bilateral hearing loss and tinnitus and visited an otolaryngologist. She was diagnosed as 
having bilateral otitis media with effusion (OME) and underwent tympanostomy tube (TT) insertion in both eardrums. However, her hearing loss did not improve after the procedure. Her bone conduction thresholds gradually worsened, and she visited our department 5 months after the onset.

Otorrhea was observed on both sides through TT. On pure-tone audiometry (Fig.1), the right ear exhibited profound deafness, and the left ear had severe mixed hearing loss. Although PR3-ANCA was negative, MPOANCA was elevated at a level of $140 \mathrm{U} / \mathrm{mL}$ in the serum. Based on these findings, she was diagnosed with OMAAV 6 months after the disease onset, according to the diagnostic criteria developed by us. ${ }^{1)}$ She had no other severe systemic symptoms at this point. Her hearing loss progressed to bilateral total deafness approximately 7 months after the onset. Along with the progression of hearing loss, lung dysfunction was revealed. Temporal bone computed tomography (CT) (Fig.2) revealed soft tissue density (STD) from the tympanum to the mastoid cavity on the left, and a slight STD in the right tympanum. No obvious cochlear ossification was observed. The T1 contrastenhanced MRI (Fig.3) revealed apparent contrast effects at both cochleae, 
indicating intracochlear bleeding and/or the destruction of the bloodlabyrinthine barrier.

Treatment with 50-mg/day prednisolone (PSL) and 50-mg/day azathioprine (AZP) was initiated from 7 months after the disease onset. Although lung dysfunction gradually improved, both deafness and tinnitus did not improve even after MPO-ANCA became negative, and the patient could not obtain satisfactory results with hearing aids. The patient underwent right CI 1 year and 10 months after the onset, because her left eardrum had perforation and CT showed STD in the left mastoid cavity.

During surgery, all electrodes could be inserted because the cochlea was not obstructed due to ossification or granulation. Her hearing ability at 9 months after the first switch-on (2 years and 7 months after the disease onset) was not good; In silent field, she could correctly identify $8 \%$ of words and $3 \%$ of sentences with auditory data alone, and $60 \%$ of words and $52 \%$ of sentences with both auditory and visual data.

Case 2: 35-year-old female

The patient had no history of otitis media, hearing loss, or trauma, 
and no family history of hearing loss. The patient was apparently well until the day before she visited our clinic, when she woke up in the morning with sudden bilateral severe hearing loss and dizziness without any trigger. On initial examination in our clinic, she had a high fever of $38.7^{\circ} \mathrm{C}$ and otitis media in both ears (Fig. 4). No obvious neurologic symptoms or nuchal rigidity were noted. The auditory brainstem response (ABR) was negative. The puretone hearing test revealed total deafness in both ears. Cerebrospinal fluid examination was negative for meningitis.

Temporal bone CT revealed STD mainly in the anterior epitympanum (Fig. 5), but the mastoid cavity was nearly normal without STD. No obvious cochlear ossification was observed. In the T1 contrast-enhanced MRI (Fig. 6), the contrast enhancement was identified in the inner ear on both sides, indicating intracochlear bleeding and/or the collapse of the blood labyrinthine barrier.

Blood examination revealed MPO-ANCA levels of $47 \mathrm{U} / \mathrm{mL}$ and PR3ANCA levels of $85 \mathrm{U} / \mathrm{mL}$, both of which were strongly positive. According to the diagnostic criteria developed by us, ${ }^{1)}$ OMAAV diagnosis was made on the 
third day after the onset. We immediately began administering steroid pulse therapy (1000-mg/day methylprednisolone for 3 days) and tacrolimus (FK506; 1-mg/day). Approximately 2 months after the onset, her general conditions gradually improved, but her hearing did not recover at all. Subsequently, we consulted with an immunologist and performed CI in her left ear at 4 months after the onset. All electrodes were inserted without any resistance due to ossification or granulation. At the first switch-on 2 weeks after surgery, she found it difficult to discriminate between sounds and gradually tended to rely on reading lips. Although discrimination between sounds improved in 1 month after the switch-on, impedance gradually increased to a level at which she could not obtain satisfactory hearing with CI.

At 3 months after the switch-on, facial nerve stimulation occurred at a level less than the maximum comfortable level, and her hearing ability gradually worsened. Eighteen months after surgery, her hearing ability was poor even with lip reading, with $18 \%$ recognition of monosyllables, $40 \%$ of words and $40 \%$ of sentences. 
Case 3: 49-year-old female

The patient had visited an otolaryngology clinic with bilateral hearing loss and otalgia 1 month before visiting our clinic. She was diagnosed as having bilateral OME, which did not improve with conservative therapy for several days, and bone conduction threshold had gradually worsened along with otalgia. The administration of steroids and antibiotics caused only temporary improvement. Eventually, otalgia and hearing loss in the left ear aggravated accompanying left facial palsy, and the patient was subsequently transferred to our clinic.

Middle ear effusion in both ears as well as moderate-degree left facial palsy was observed. Pure-tone audiogram revealed bilateral severe mixed hearing loss (Fig. 7). In the temporal bone CT, the mastoid air-cell growth and aeration were good, but bilateral epi- and mesotympanum were filled with STD. The blood examinations revealed mild inflammation (WBC, 7800×103/ul,; CRP, $1.12 \mathrm{mg} / \mathrm{dL})$, but positive PR3-ANCA (12.5 U/mL). Chest CT revealed no lung nodules.

According to the criteria developed by us, ${ }^{1)}$ we diagnosed with OMAAV 
in 2 months after the disease onset and initiated remission induction therapy with 60-mg/day PSL and 100-mg/day cyclophosphamide (CY). Three months after the disease onset, her hearing recovered almost to the normal range. However, 6 years and 7 months after the onset, bilateral bone conduction gradually worsened. although she showed clear eardrums, no abnormalities on temporal bone CT and negative PR3-ANCA. She did not respond to the same treatment any more, and eventually became totally deaf in both ears 7 years after the disease onset. The patient underwent $\mathrm{CI}$ in the right ear 7 years and 5 months after the disease onset. We found slight granulation tissue in the mastoid during the CI operation. All electrodes were inserted without any resistance by ossification nor granulation. Her hearing ability was very good after surgery, with $90 \%$ recognition of monosyllables, $100 \%$ recognition of words, and $100 \%$ recognition of sentences. One year after CI, she got dizziness and her blood examinations revealed positive PR3-ANCA (8.6 $\mathrm{IU} / \mathrm{ml})$ at the same time.

Case 4: 67-year-old female

The patient has had no history of otitis media and head trauma, and 
no family history of hearing loss. Six months before visiting our hospital, she suddenly recognized bilateral hearing loss, and visited an otolaryngologist. She was diagnosed as having bilateral OME, and underwent TT insertion on both sides. However, her hearing did not improve after the procedure, and her bone conduction thresholds gradually worsened. She became totally deaf on both sides 4 months after the onset, and she visited our clinic 6 months after the onset. Pure-tone audiogram revealed bilateral total deafness. In the temporal bone CT, the mastoid air-cell growth and aeration were moderate, but STD was seen in bilateral epi- and mesotympanum. No obvious cochlear ossification was observed. The blood examinations revealed mild inflammation (WBC, $8900 \times 10^{3 / \mu l}, \cdot \mathrm{CRP}, 2.78 \mathrm{mg} / \mathrm{dL}$ ) and positive MPOANCA (45.7 U/mL). Chest CT revealed no lung nodules. The T1 contrastenhanced MRI (Fig.8) revealed apparent contrast effects at both cochleae, indicating intracochlear bleeding and/or the destruction of the bloodlabyrinthine barrier.

According to the criteria developed by us ${ }^{1)}$, we diagnosed with OMAAV in 6 months after the disease onset, and initiated remission induction 
treatment with steroid pulse (1000-mg/day methylprednisolone for 3 days) and methotrexate (MTX; 8-mg/week) from 7 months after the disease onset. But her hearing did not improve even after MPO-ANCA became low (3.60 $\mathrm{U} / \mathrm{mL}$ ). We performed $\mathrm{CI}$ in her left ear at 8 months after the onset. No ossification or granulation tissue was observed in the cochlea during surgery, and all electrodes could be inserted. At the first switch־on of the CI 2 weeks after surgery, she could feel the sounds, but she could not discriminate languages; her hearing ability were very poor with $0 \%$ recognition of monosyllables, words and sentences.

Characteristic features of all patients undergoing CI for deafness due to OMAAV including those reported in the other literature are summarized in Table 1. Five of six patients are females. The time from the onset of hearing loss to CI varied ranging from 4 to 71 months, and it was noted that the prognosis of hearing after CI was poor in patients with short interval from the onset to total deafness (our cases 1, 2, and 4). Serum ANCA was positive in the majority of patients, and both MPO- and PR3-ANCA was positive in one patient (our case 2). Other involvements and symptoms included lung, 
orbit, and facial palsy. Agents used for their treatment were mainly steroids and immunosuppressive agents.

\section{Discussion}

To date, five patients, including our present four patients who underwent CI due to deafness caused by OMAAV have been reported. In three of them, hearing loss progressed gradually to total bilateral deafness during the treatment of polyangiitis granulomatosis, whereas in the other three patients, bilateral total deafness occurred rapidly before initiating treatment. Regarding serum ANCA, it was positive in the patient reported by AbouElhmd et al.,5) and were also positive in all four of our patients; particularly in our case 2, both MPO and PR3-ANCA were positive (Table 1).

It was noted that the CI outcome of case 3 , who exhibited slow progression of hearing loss, was good, whereas that of the other three patients with rapid progression of hearing loss was poor; bilateral deafness occurred on the day of the disease onset in case 2 and at 7 months and 6 months after the onset in cases 1 and 4, whereas it occurred at 7 years after the onset in 
case 3. It is also possible that progressive hearing loss in case 3 might be cause by other factors than OMAAV since her PR3-ANCA remained negative and only slight granulation was observed in the mastoid during CI surgery. One of the important pathological factors of OMAAV is vasculitis, and it appears quite possible that the pathogenesis of hearing loss in this disease is closely related to circulatory problems caused by vasculitis. Most of the structures in the cochlea are supplied by branches of the main cochlear artery. The stria vascularis is supplied by its small branch via the external radiating arteriole, and the spiral ganglion is supplied by the spiral modiolar artery as well as by a small branch separating from the internal radiating arteriole.6) Furthermore, the stria vascularis is one of the farthest target organs in the cochlea from the main cochlear artery in terms of blood supply. Studies have reported that the diameter of the vessels in the stria vascularis is $7-15 \mu \mathrm{m}$, that of the spiral modiolar artery is $10-20 \mu \mathrm{m}$, and that of the labyrinthine artery, which supplies nerves to the internal auditory canal as well as inner ear, is as large as 200-900 $\mu \mathrm{m} .7,8)$. Considering this, it does not appear contradictory that organs, such as the stria vascularis, which are supplied by 
small blood vessels running for a longer distance, may easily be affected by AAV.9) In other words, only vessels supplying the stria vascularis might have been affected in the early stage in mild cases, such as case 3 in the present study, and this may agree with the fact that hearing loss recovered temporarily with initial treatments including steroids. However, as the disease progressed, hearing loss may have progressed gradually owing to extended involvement of blood vessels, including the labyrinthine artery, in the order from small to large. In case 3, we speculated that the spiral ganglion cells had remained intact.

On the other hand, in cases 1,2 , and 4 , it might be inferred that not only vessels supplying the stria vascularis, but also the other important inner ear vessels were rapidly impaired, as was revealed by contrast-enhanced MRI, indicating that the blood-labyrinthine barrier might be destroyed and may have affected cochlear nerve as well. These findings support the fact that the efficacy of CI was poor in them. Thus, in patients with OMAAV, it might be inferred that labyrinthine ischemia and inflammation affects various structures, such as the stria vascularis, hair cells, and spiral ganglia, within 
the inner ear, causing several symptoms and marked histories.

The cochleas were clearly enhanced in contrast-enhanced MRI study in cases 1,2 , and 4 , indicating leakage of the contrast medium out of the blood vessels and severe destruction of the blood-labyrinthine barrier in the inner ear, such as inner ear bleeding ${ }^{10-12)}$. Because we did not have contrastenhanced MRI study in case 3, whose performance with CI was good, we did not confirm that the enhancement in the cochlea during contrast-enhanced MRI study is a specific finding predicting poor efficacy of CI. However, it seems quite possible that such severe findings of contrast-enhanced MRI of the cochlea indicating intracochlear bleeding may be another predictor of the inefficacy of CI in patients with OMAAV as well as that of the history of rapid progression of hearing loss.

Intracochlear ossification or granulation formation was observed during CI surgery in no patient including our four patients, on whom labyrinthitis or labyrinthine bleeding were likely to occur according to MRI findings. Although it is unknown whether such pathology is generally rare in patients with OMAAV, it may indicate that the labyrinthine pathology in 
OMMAV, possibly comprising of vasculitis, ischemia, and necrosis, is different from that in bacterial labyrinthitis, which occasionally causes osteitis of the otic capsule as well. This may be consistent with the fact that these intracochlear findings during surgery were not compatible with poor efficacy of $\mathrm{CI}$ in cases 1,2 , and 4 .

It is a difficult question whether a patient with rapidly progressing bilateral deafness due to OMAAV is a candidate for CI. Cases 1 and 2, where CI was ineffective, actually have difficulty in listening only with the CI, but they can talk with each other by reading lips. Thus, we suggest that CI improves the quality of life even in such patients, although the improvement is limited. The effectiveness of CI for patients with hearing loss due to OMAAV does not appear satisfactory, but it may be considered as a favorable treatment option if the vasculitis is systemically well controlled.

All of our four cases were females. However, our analysis of all reported patients with OMAAV indicated that hearing loss by OMAAV has no significant sexual difference ${ }^{1}$. Although there might be some factor predisposing female patients with OMAAV to severe or profound bilateral 
hearing loss, we have not yet obtained any evidence of it.

\section{Conclusions}

We have reported four patients undergoing CI for bilateral deafness caused by OMAAV. In three of them, with histories of hearing loss being more rapid than the remaining patient, outcomes after CI were poor. The inner ears were enhanced in contrast-enhanced MRI study in these patients. The results were attributed to the possible extension of vascular lesion not only to the stria vascularis and hair cells of the cochlea but also to the spiral ganglion. Therefore, we suggest that preoperative evaluation of history of hearing loss as well as the findings of contrast-enhanced MRI may be important for predicting the prognosis of CI. Although the efficacy of CI was considerably variable, it can be considered as a treatment option for patients with deafness due to OMAAV.

Conflicts of interest

The authors declare that they have no conflict of interest

Acknowledgments 
References

1) Harabuchi Y, Kishibe K, Tateyama K, Morita Y, Yoshida N,Kunimoto Y,et al.:Clinical features and treatment outcomes of otitis media with antineutrophil cytoplasmic antibody (ANCA)-associated vasculitis (OMAAV) : a retrospective analysis of 235 patients from a nationwide survey in Japan.

Mod Rheumatol 2017;27:87-94

2) Yoshida N, Iino Y. Pathogenesis and diagnosis of otitis media with ANCA-associated vasculitis. Allergol Int 2014;63:523-532.

3) Yoshida N, Kishibe K, Tateyama K, Okada M, Sakaguchi H, et al.:Clinical features of 90 cases of otitis media with ANCA associated vasculitis(In Japanese). Otol Jpn 2014;24:53-61.

4) Yoshida N, Hara M, Hasegawa M, Matsuzawa S, Shinnabe A, Kanazawa H,et al.: Reversible cochlear function with ANCA-associated vasculitis initially diagnosed by otologic symptoms. Otol Neurotol 2014;35:114-120.

5) Abou-Elhmd KA, Hawthorne MR, Flood LM.: Cochlear implantation 
in a case of Wegener's granulomatosis: J Laryngol Otol $1996 ; 110: 958-961$.

6) Schuknecht H, Chapter 7, Vascular anatomy, Anatomy of the Temporal Bone with Surgical Implications, ed by Schuknecht H and Gulya AJ, Lee \& Febiger, Philadelphia 1986;202-207.

7) Nomura Y, Hiraide F. : Cochlear blood vessel. A histochemical method of its demonstration. Arch Otolaryngol 1968;88:231-237.

8) Kirikae I, Nomura Y, Hiraide F: The capillary in the human cochlea. Acta Otolaryngol 1969;67:1-8.

9) Jennette JC, Falk RJ, Andrassy I, Bacon PA, Churg J, Gross WL, et al.:Nomenclature of systemic vasculitides. Proposal of an international consensus conference. Arthritis Rheum 1994;37:187-92.

10) Kastenbauer S, Klein M, Koedel U, Pfister HW: Reactive nitrogen species contribute to blood-labyrinth barrier disruption in suppurative labyrinthitis complicating experimental pneumococcal meningitis in the rat. Brain Res 2001;904: 208-17.

11) Sugiura M, Naganawa S, Teranishi M, Sato E, Kojima S, Nakashima 
T: Inner ear hemorrhage in systemic lupus erythematosus.

Laryngoscope 2006;116:826-828

12) Yoshida T, Sugiura M, Teranishi M, Nakata S, Nakashima T:

Breakdown of the Blood-labyrinth Barrier in Sensorineural hearing loss (In Japanese). Audiol Jpn 2007;57:152-162. 
Figure legends

Fig. 1.

Pure-tone audiometry of case 1 at the first visit to our clinic Fig. 2.

Temporal bone CT of case 1 . On the left ear, her tympanum and mastoid cavity were filled with soft tissue density (arrows). Rt: right ear, Lt: left ear.

Fig. 3.

Contrast-enhanced MRI of case 1. Contrasting effect in the bilateral cochlea was observed (arrows). Rt: right ear, Lt: left ear. Fig. 4.

Right (Rt) and left (Lt) eardrums of case 2, showing hyperemia and bullae formation

Fig. 5.

Temporal bone CT of case 2. There is soft tissue density (arrows) in the anterior epitympanum. Rt: right ear, Lt: left ear. Fig. 6. 
Contrast-enhanced MRI of case 2. Contrasting effect in the bilateral cochlea was observed (arrows). Rt: right ear, Lt: left ear. Fig. 7.

Pure-tone audiometry of case 3 at the first visit to our clinic Fig. 8.

Contrast-enhanced MRI of case 4. Contrasting effect in the bilateral cochlea was observed (arrows). Rt: right ear, Lt: left ear. 
Table 1. Comparisons of five cases with cochlear implantation due to deafness caused by OMAAV

\begin{tabular}{|l|l|l|l|l|l|c|c|}
\hline Author & Age & Sex & $\begin{array}{l}\text { Time from } \\
\text { onset to CI }\end{array}$ & $\begin{array}{l}\text { Serum } \\
\text { ANCA }\end{array}$ & $\begin{array}{l}\text { Contrast } \\
\text { enhance on MRI }\end{array}$ & $\begin{array}{l}\text { Intracochlear } \\
\text { ossification }\end{array}$ & Prognosis \\
\hline $\begin{array}{l}\text { Abou-Elhmd, } \\
\text { et al. (1996) }\end{array}$ & 71 & female & 24 months & + & yes & no \\
\hline Case 1 (2015) & 71 & female & 22 months & MPO + & yes & poor \\
\hline Case 2 (2013) & 35 & female & 4 months & $\begin{array}{l}\text { MPO }+ \\
\text { PR3 }+\end{array}$ & PR3 + & no & good \\
\hline Case 3 (2014) & 49 & female & 89 months & no & no & poor \\
\hline Case4 (2016) & 76 & female & 8 months & MPO + & yes & & \\
\hline
\end{tabular}




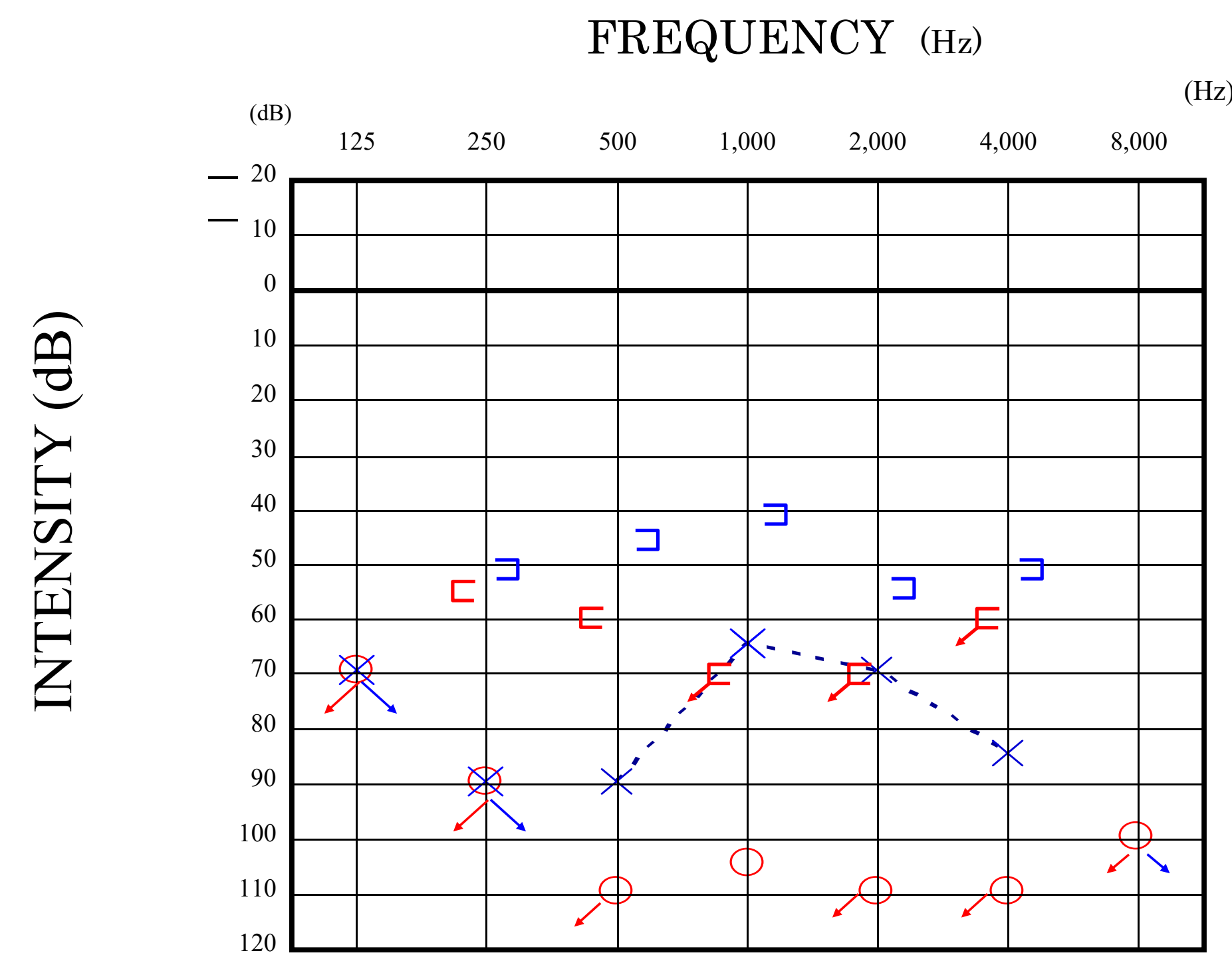

Fig. 1. 


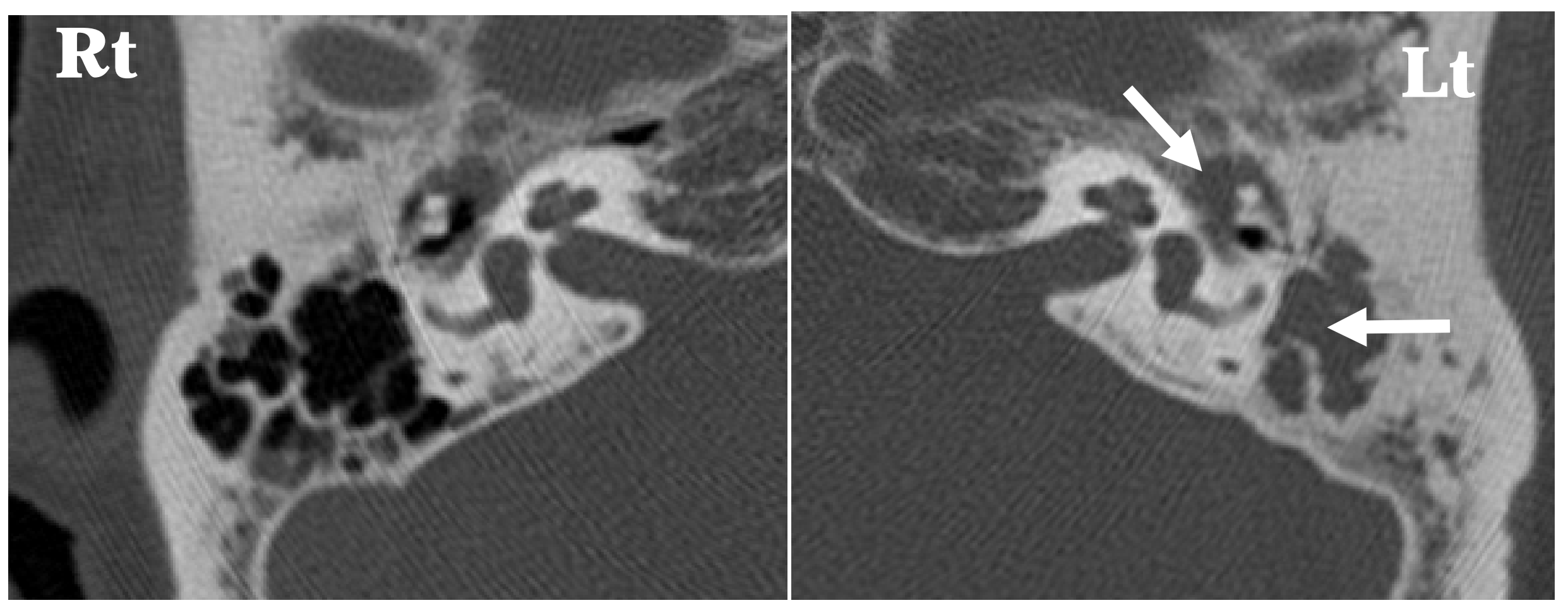

Fig. 2. 


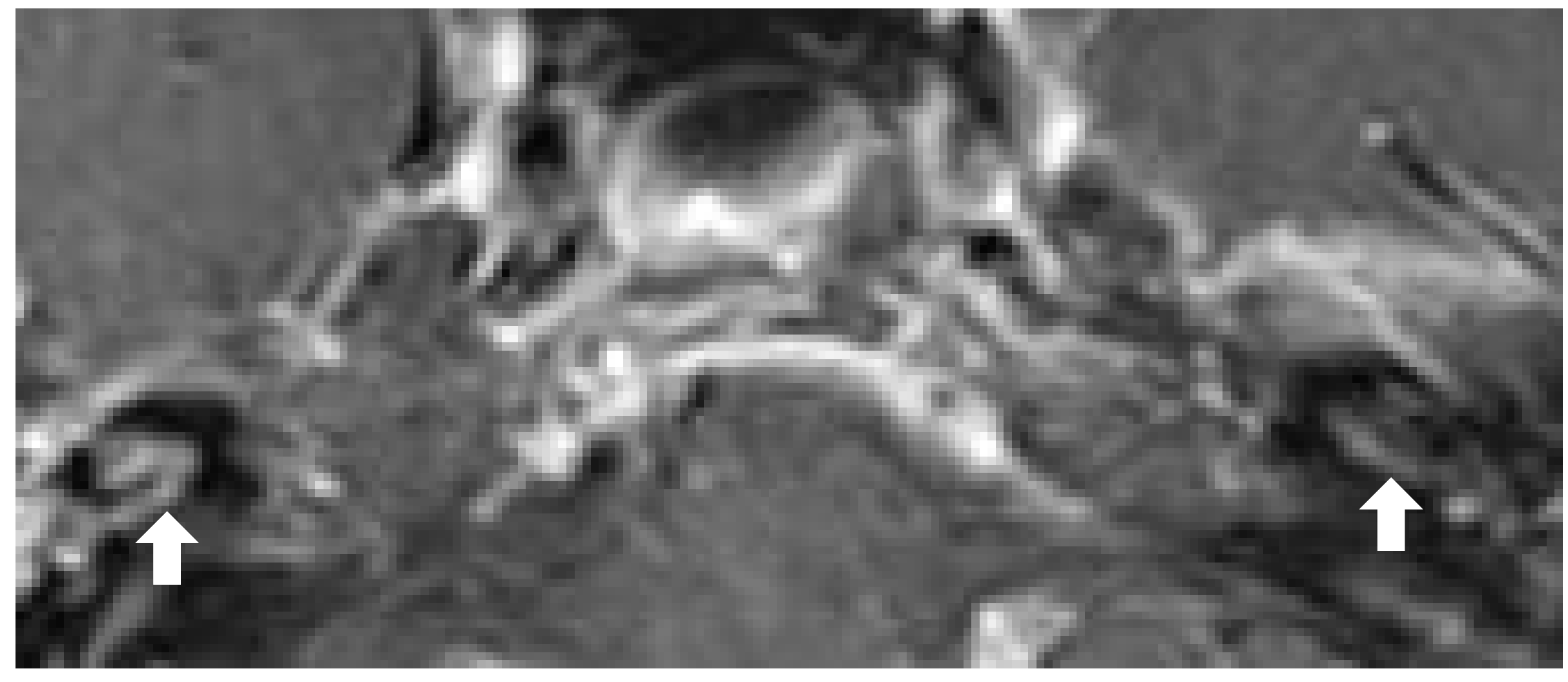

Fig. 3. 

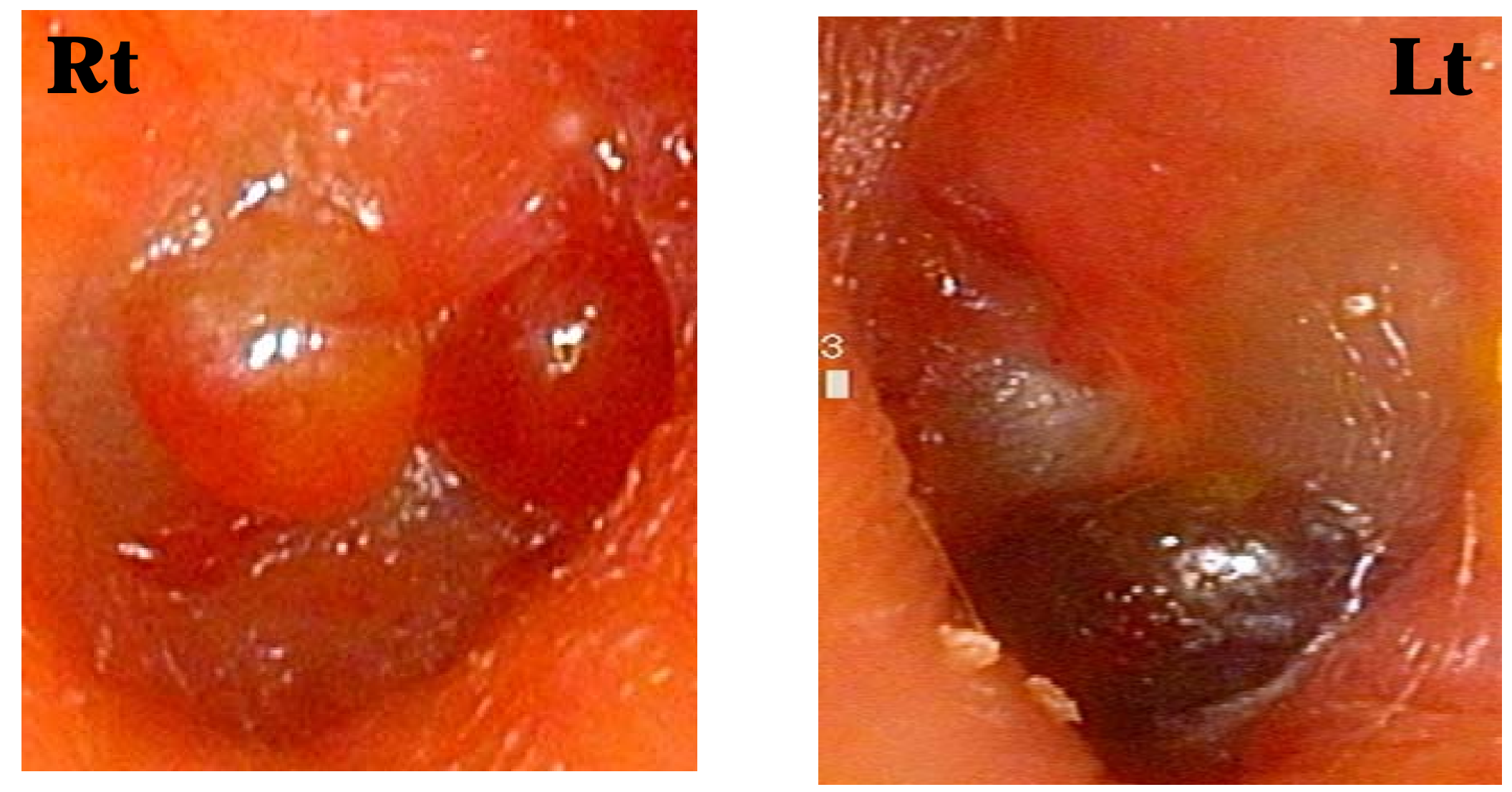

Fig. 4. 


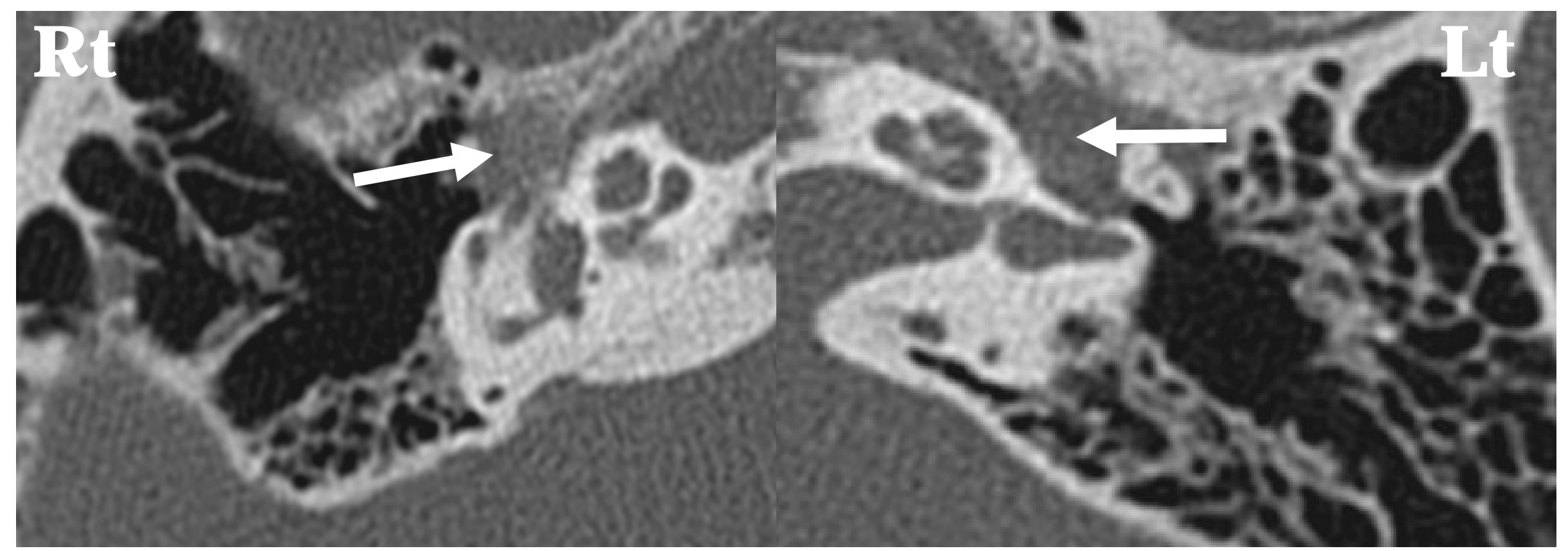

Fig. 5. 


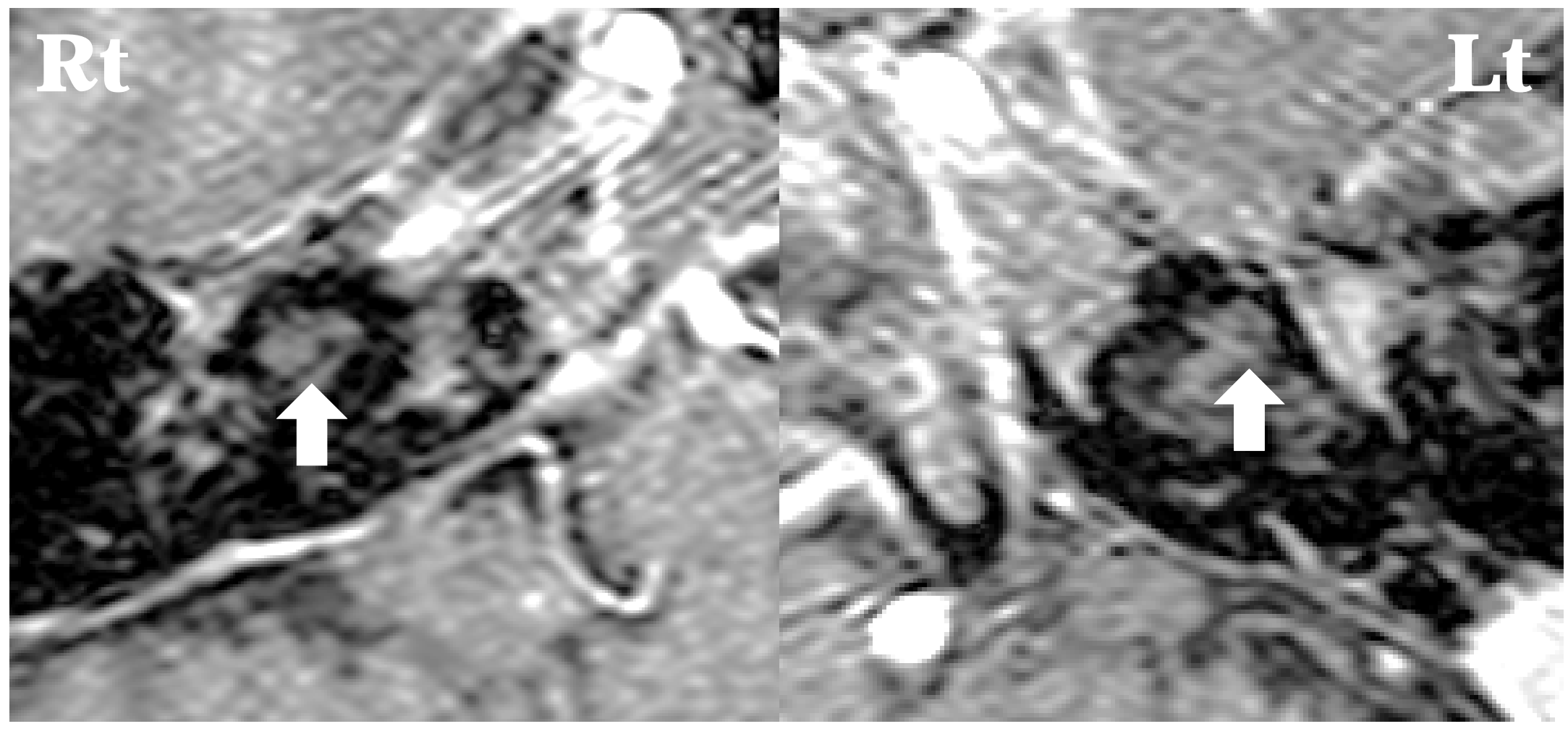

Fig. 6. 


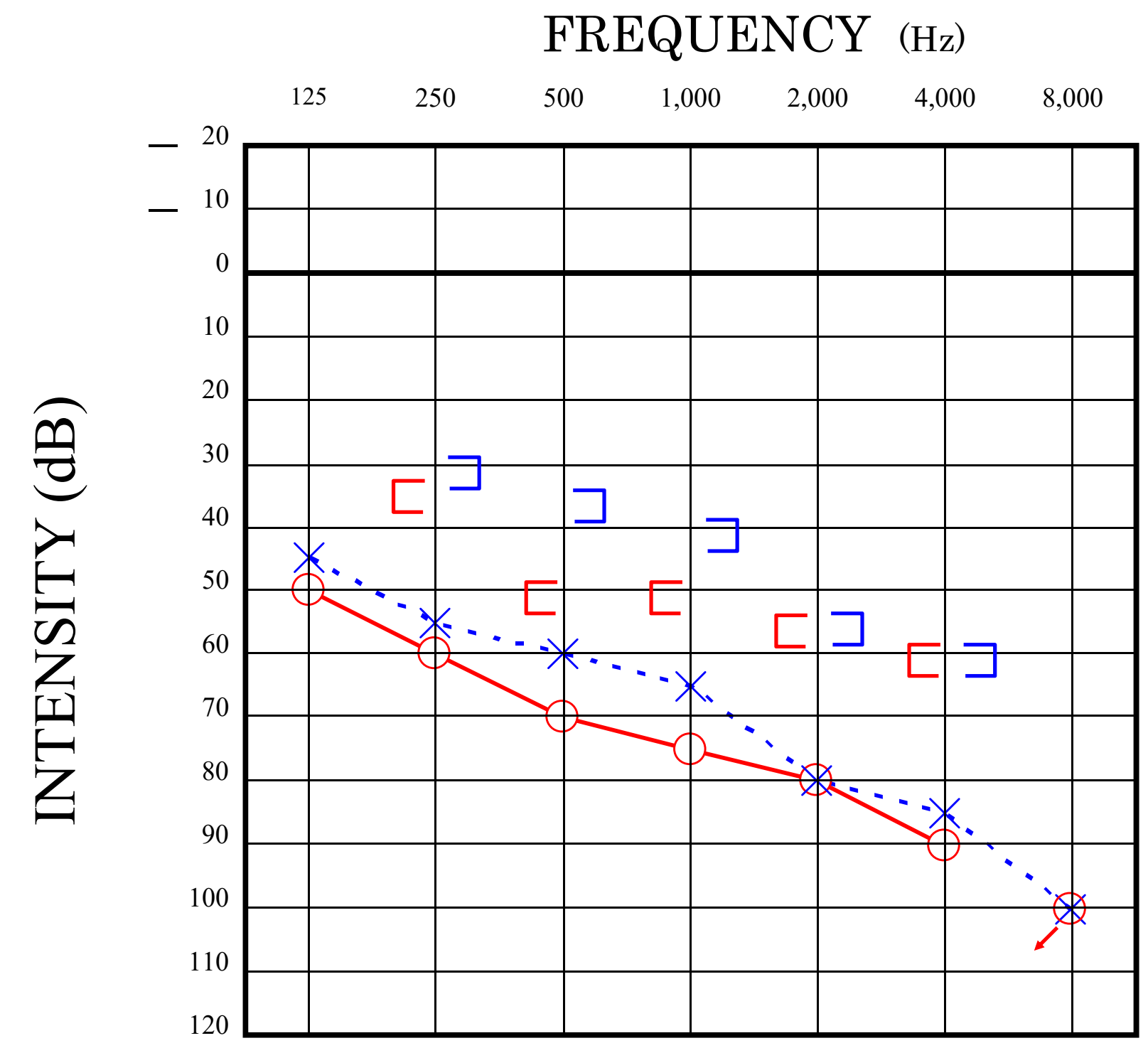

Fig. 7. 


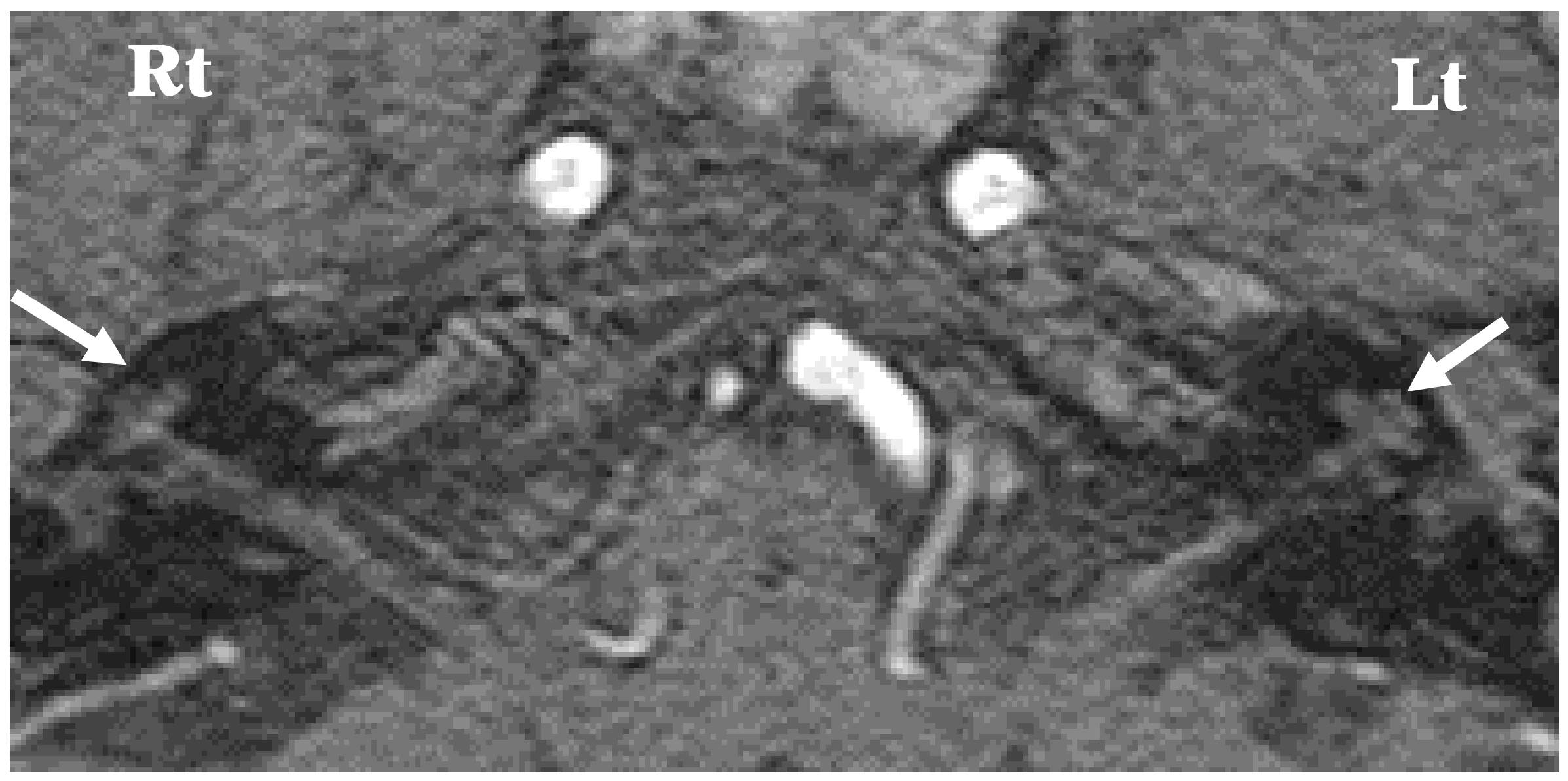

Fig.8 